\title{
30 years of immunomodulation by intravenous immunoglobulin
}

\section{"Despite the difficulty in defining the multiple and complex mechanisms of action of intravenous immunogobulin, this biological drug - be it transiently or for long-term benefit - became essential for many patients..."}

\section{KEYWORDS: immune disturbance $=$ immune thrombocytopenia " immunomodulation - intravenous immunoglobulin}

Since 1946, fractionation of human plasma or serum into proteins and lipoproteins by ethyl alcohol [1], and later on extraction of gammaglobulin from other plasma components by low $\mathrm{pH}$ [2], led to subcutaneous, substitutive gammaglobulin treatment in patients with primary or secondary hypogammaglobulinemia.

The anticomplementary activities of aggregates of gammaglobulin lead to severe side effects in some patients [3]. Although such side effects were reduced by the enzymatic treatment of gammaglobulins with pepsin, trypsin, plasmin digestion or by chemical modifications, such administered preparations were rapidly removed from the circulation by the reticuloendothelial system.

Further purification of gammaglobulins at acidic $\mathrm{pH} 4$ and additional procedures resulted in an unmodified immunoglobulin for intravenous administration named intravenous immunogobulin (IVIG) and characterized by its unimpaired biological functions [4].

Today, IVIG is extracted from the pooled plasma of 10,000-60,000 blood or plasma donations. It contains several million antibody specificities, mainly natural and anti-idiotypic antibodies [5]. The safety of the IVIG product is provided by careful selection of donors, viral inactivation and validation of each production step [6]. Tolerability depends on the different IVIG products [6]. Adverse effects of IVIG occur in 5\% of recipients and resemble symptoms similar to a viral infection (e.g., headache, chills, myalgia, nausea and fever). Severe side effects (aseptic meningitis or thromboembolic events with or without stroke) are rare [6].

\section{Immune thrombocytopenia as a model of immunomodulatory effects of IVIG}

The impetus to start IVIG treatment in immune thrombocytopenia (ITP) came from a boy with
Wiskott-Aldrich syndrome, a rare recessive $\mathrm{X}$-linked disease with hypogammaglobulinemiaassociated infections and thrombocytopenia. After periodic substitutive IVIG treatment, his moderate thrombocytopenia always showed a slight platelet increase. In 1980, we started the first IVIG administration of $0.4 \mathrm{~g} / \mathrm{kg}$ bodyweight in a boy with long-term severe bleedings caused by ITP and with secondary hypogammaglobulinemia due to long-term immunosuppressive treatment. After the first administration of IVIG, his platelet counts dramatically increased and we continued the same dose for a total of 5 days. In a pilot study, 12 consecutive children with ITP and platelet counts below $20 \times 10^{9}$, but without hypogammaglobulinemia, showed the same phenomena [7]. In parallel, we treated two children with aplastic anemia with the same schedule of IVIG and did not observe any platelet increase.

To explain why we began this new treatment, as a young clinician at the children's hospital in Lucerne, Switzerland I actively participated in Jürg Sartorius' first randomized study 'corticosteroids versus observation' in children with ITP in 1975 in Basel, Switzerland. This study convinced me that ITP is a phenomenon of a postinfectious immune disturbance in some predisposed children. Moving to Bern, Switzerland and working at the Institute of Experimental Cancer Research (Drs Barandun, Morell and Wagner), I was able to discuss immunology in depth. A year later I was asked to join the University Children's Hospital, Insel Hospital, Bern, where I was able to start IVIG studies together with my collegues. When Sartorius died of cancer, as a 40 year old I was asked to consult his Pediatric HematologyOncology division at the University Children's Hospital in Basel. There I met Dr E Kleihauer, a well-known pediatric hematologist from

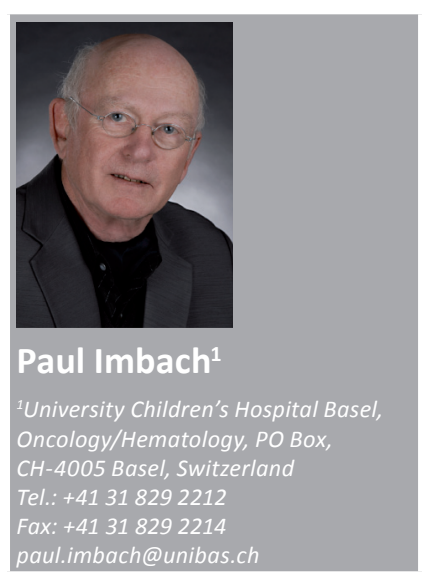

Future :
Medicine $\%$ part of 
Ulm, Germany. We discussed our early experiences with IVIG in ITP. He enthusiastically encoureged me to continue our studies.

Soon, this new finding of IVIG was confirmed in adult patients with ITP in parallel to our controlled, randomized, multicenter study in children $[8,9]$. Retrospectively, a few years before that, two reports - one on a child with ITP and varicella, and another on two adults with hypogammaglobulinemia and thrombocytopenia - were recognized, who showed an increase of their platelet counts after substitutive gammaglobulin administration.

The possible mechanisms of action of IVIG in the rare autoimmune disorder ITP described in the first report [7] evoked huge clinical and laboratory research in many other inflammatory and autoimmune diseases with a similar immune pathogenesis. This pathogenesis is characterized by antibodies or immune complexes triggering a state of hyper- and/or altered immune responses in susceptible individuals.

“...administration of IVIG synergistically modulates the disturbed immune response through the innate and the adaptive immunity: from dendritic cells to $T$ and $B$ cells to the effector cells of phagocytosis and their specific molecules..."

More than 500 peer-reviewed articles are indexed in Pubmed on the mechanisms of action of IVIG - alongside over 30,000 articles on the clinical effects of IVIG. The main conclusion of these articles is that administration of IVIG synergistically modulates the disturbed immune response through the innate and the adaptive immunity: from dendritic cells to T- and B-cells to the effector cells of phagocytosis and their specific molecules such as cytokines, receptors, ligands, complement and and other components [10].

The main elements of the mechanisms of actions of IVIG administration are:

- On the level of macrophages/phagocytosis: blockade of Fc receptors of the reticuloendothelial system, mainly in the spleen [8]. Later on, Ravetch's group demonstrated stimulation of the inhibitory Fc $\gamma$ receptor IIB on macrophages [11];

- On B cells: neutralization of specific autoantibodies by anti-idiotypic antibodies of IVIG, downregulation of autoantibody production $[12,13]$ and neutralization of B-cell survival and activating factors such as BAFF and the proliferation-inducing ligand APRIL [14].
- On T cells: IVIG modulates T-cell reactivity [15], cytokine release and increases Tregs [16]. Recently, it was reported that the differentiation and amplification of the Th17 cells and their effector cytokines are inhibited by IVIG [17];

- On complement: attenuation of complement activation by IVIG has been reported in complement-dependent autoimmune diseases [18,19];

- On apoptosis: anti-Fas antibodies may influence apoptosis [20];

- On dendritic cells: in the last decade the modulation and the functions of dendritic cells by IVIG have been demonstrated, first by Bayry et al. [21]. Adoptive transfer of IVIG-primed splenic leucocytes (CD11c dendritic cells) to thrombocytopenic mice is a fascinating approach. The administered dose of the adoptive transfer of IVIG-primed cells to thrombocytopenic mice was dramatically lower in comparison with IVIG treatment [22]. Thus, IVIG might form soluble immune complexes on dendritic cells and induce regulatory activities on the disturbed immune response. Such a model might be studied in humans. However, considering the cost involved in dendritic cell therapy, such an approach might be a daunting task.

The majority of laboratory research on the mechanisms of action of IVIG are based on murine models. They seem to be useful in understanding the immunomodulatory effects; however, direct translation to IVIG effects in patients remain critical. Thus, the exact immunomodulatory effects still remain incompletely understood (for detailed reviews, see $[10,23])$.

\section{Immunomodulatory treatment with IVIG}

The indications of IVIG are seldomly based on evidence-based clinical trials. The heterogeneity of autoimmune diseases, their various clinical manifestations and their complex alterations of the immune responses are the main obstacles of evidence-based indications. These facts are the reasons for the frequent off-label use of IVIG. Today, the main indications of IVIG are:

- In the field of neurology: Guillain-Barré syndrome, chronic inflammatory demyelinating polyneuropathy and multifocal motor neuropathy; 
- In dermatology: toxic epidermal necrolysis, Steven-Johnson syndrome and the different forms of pemphigus. In the latter, a combination of IVIG and rituximab in repetitive courses of 5-6 months showed long-term benefits in patients with severe blistering disease;

- In hematological autoimmune cytopenias: firstline treatment with IVIG are for patients with bleeding in ITP, fetal and neonatal alloimmune thrombocytopenia, post-transfusion purpura and neonatal hemochromatosis in pregnant women.

- In other immune-related disorders: Kawasaki disease and transplantations. Some reports demonstrate convincing results with IVIG for recurrent spontaneous abortion, while others are critical. Clinical trials are ongoing and promising in early-stage Alzheimer's disease.

\section{Financial aspects of IVIG \& alternative treatment modalities}

The total world consumption of IVIG currently exceeds 100 tons per year according to International Blood/Plasma News (Marketing Research Bureau Inc., CA, USA). In this context, it is difficult to understand why IVIG still remains so costly. Certainly, the procedure from the source (donations of blood/plasma) according to good manufacturing procedures and controls, the professional distribution as well as the more precise indications beside the off-label uses and their surveillance are expensive. The high amounts of use compared with the limited resource may be one of the reasons for not reducing the high price.

Modulation of IVIG into glycoforms such as sialylated Fc fragments may be an alternative to the pure IVIG in the future. This recombinant sialylated IgG Fc fragment enhances the expression of the inhibitory $\mathrm{Fc} \gamma$ receptor IIB on effector macrophages (summarized in [24]).

In ITP, the new thrombopoitin receptor agonists with evidence-based clinical studies may provide an alternative to IVIg - at least in the

\section{References}

1 Cohn EJ, Strong LE, Hughes WL et al. Preparation and properties of serum and plasma proteins; a system for the separation into fractions of the protein and lipoprotein components of biological tissues and fluids. J. Am. Chem. Soc. 68, 459-475 (1946).

2 Oncley JL, Melin M. The separation of the antibodies, isoagglutinins, prothrombin, plasminogen and beta1-lipoprotein into subgroup of patients with the need to prevent the risk of severe, chronic ITP bleedings [25]. These agonists stimulate platelet production in megakarycoytes and the platelet increase occur 3-7 days after the first administration of the drug.

\section{Future perspective}

The clinical research focuses on the early stage of a chronic inflammatory or autoimmune disease where the immune response is not definitively overwhelmed. However, often the heterogeneity, stage and progress of a disease is far from being clear. Therefore, clinical registries with huge numbers of patients with the same diagnosis are necessary before doing evidence-based studies. The aim of a registry is to elucidate the natural history of ITP (or of other diseases) and to distinguish subgroups of an entity (i.e., [101]). On such subgroups, controlled clinical and laboratory studies including genetic analyses may become feasible.

\section{Conclusion}

Reestablishment of immune homeostasis is the aim of treatment with human IVIG. Despite the difficulty in defining the multiple and complex mechanisms of action of IVIG, this biological drug - be it transiently or for long-term benefit - became essential for many patients with an inflammatory or an autoimmune disease, especially for those patients with no equivalent alternative treatment.

\section{Acknowledgements}

The author thanks Jagadeesh Bayry for his reading of and corrections to this manuscript.

\section{Financial \& competing interests disclosure}

$P$ Imbach has received Institutional support from CSL Behring, Amgen and GlaxoSmithKline. The author has no other relevant affiliations or financial involvement with any organization or entity with a financial interest in or financial conflict with the subject matter or materials discussed in the manuscript apart from those disclosed. No writing assistance was utilized in the production of this manuscript. subfractions of human plasma. J. Am. Chem. Soc. 71(2), 541-550 (1949).

3 Barandun S, Kistler P, Jeunet F, Isliker H. Intravenous administration of human gammaglobulin. Vox. Sang. 7, 157-174 (1962).

4 Kistler P, Nitschmann H. Large scale production of human plasma fractions. Eight years experience with the alcohol fractionation procedure of Nitschmann, Kistler and Lergier. Vox. Sang. 7, 414-424 (1962).
5 Seite JF, Shoenfeld Y, Youninou P, Hillion S. What is the contents of the magic draft IVIG? Autoimmun. Rev. 7, 435-439 (2008).

6 Hooper JA. Intravenous immunoglobulins: evolution of commercial IVIG preparations. Immunol. Allergy Clin. North Am. 28, 765-778 (2008).

7 Imbach P, Barandun S, d'Apuzzo V et al. High-dose intravenous gammaglobulin for idiopathic thrombocytopenic purpura in childhood. Lancet 1, 1228-1231 (1981). 
8 Fehr J, Hofman V, Kappeler U. Transient reversal of thrombocytopenia in idiopathic thrombocytopenic purpura by high-dose intravenous gamma globulin. N. Engl. J. Med. 306, 1254-1258 (1982).

9 Newland AC, Treleaven JG, Minchinton RM, Waters AH. High-dose intravenous IgG in adults with autoimmune thrombocytopenia. Lancet 1(8316), 84-87 (1983).

10 Imbach P, Lazarus A, Kühne T. Intravenous immunoglobulins induce potentially synergistic immunomodulations in autoimmune disorders. Vox. Sang. 98, 385-394 (2010).

11 Samuelsson A, Towers T, Ravetch J. Anti-inflammatory activity of IVIG mediated through the inhibitory Fc receptor. Science 291, 484-486 (2001).

12 Rossi F, Kazatchkine MD. Antiidiotypes against autoantibodies in pooled normal human polyspecific Ig. J. Immunol. 143, 4104-4109 (1989).

13 Kondo N, Ozawa T, Mushiake K et al. Suppression of immunoglobulin production of lymphocytes by intravenous immunoglobulin. J. Clin. Immunol. 11, 152-158 (1991).

14 Le Pottier L, Sapir T, Bendaoud B, Youinou P, Shoenfeld Y, Pers JO. Intravenous immunoglobulin and cytokines: focus on tumor necrosis factor family members BAFF and APRIL. Ann. NY Acad. Sci. 1110, 426-432 (2007).
15 Semple JW, Freedman J. Increased antiplatelet $\mathrm{T}$ helper lymphocyte reactivity in patients with autoimmune thrombocytopenia. Blood 78(10), 2619-2625 (1991).

16 Ephrem A, Chamat S, Miquel C et al. Expansion of D4+CD25+ regulatory $\mathrm{T}$ cells by intravenous immunoglobulin: a critical factor in controlling experimental autoimmune encephalomyelitis. Blood 111, 715-722 (2008).

17 Maddur MS, Vani J, Hegde P, LacroixDesmazes S, Kaveri SV, Bayry J. Inhibition of differentiation, amplification, and function of human TH17 cells by intravenous immunoglobulin. J. Allergy Clin. Immunol. 127(3), 823-830.e1-7 (2011).

18 Lutz HU, Stammler P, Bianchi V et al. intravenously applied IgG stimulates complement attenuation in a complementdependent autoimmune disease at the amplifying C3 convertase level. Blood 103, 465-472 (2004).

19 Basta M, Van Goor F, Luccioli S et al. Metcalfe D. F(ab)'2-mediated neutralization of C3a and C5a anaphylatoxins: a novel effector function of immunoglobulins. Nat. Med. 9, 431-438 (2003).

20 Viard I, Wehrli P, Bullani R et al. Inhibition of toxic epidermal necrolysis by blockade of CD95 with human intravenous immunoglobulin. Science 282, 490-493 (1998).
21 Bayry J, Lacroix-Desmazes S, Carbonneil C et al. Inhibition of maturation and function of dendritic cells by intravenous immunoglobulin. Blood 101, 758-765 (2003).

22 Siragam V, Crow AR, Brine D, Song $S$, Freedman J, Lazarus AH. Intravenous immunoglobulin ameliorates ITP via activating Fcgamma receptors on dendritic cells. Nat. Med. 12, 688-692 (2006).

23 Negi VS, Elluru S, Siberil S et al. Intravenous immunoglobulin: an update on the clinical use and mechanisms of action. J. Clin. Immunol. 27, 233-245 (2007).

24 Anthony RM, Kobayashi T, Wermeling F, Ravetch JV. Intravenous gammaglobulin suppresses inflammation through a novel $\mathrm{T}(\mathrm{H}) 2$ pathway. Nature 475(7354), 110-113 (2011).

25 Imbach P, Crowther M. Thrombopoietinreceptor agonists for primary immune thrombocytopenia. N. Engl. J. Med. 365(8), 734-741 (2011).

\section{Website}

101 The Intercontinental Cooperative ITP Study Group. www.itpbasel.ch 